\title{
Multiple plastic versus fully covered metal stents for managing post-liver transplantation anastomotic biliary strictures: a meta- analysis of randomized controlled trials
}

\author{
Alberto Tringalia, Ilaria Tarantino ${ }^{b}$, Luca Barresi $^{b}$, Mario Traina $^{b}$, Giulia Bonato $^{a}$, Marcello Cintolo ${ }^{a}$, \\ Cesare Hassanc, Massimiliano Mutignania , Douglas G. Adler ${ }^{d}$ \\ Ospedale Ca’ Granda Niguarda, Milan, Italy; IRCCS ISMETT, Palermo, Italy; Nuovo Regina Margherita Hospital, \\ Rome, Italy; University of Utah, Salt Lake City, Utah, USA
}

Abstract

Background Anastomotic biliary strictures (ABS) following liver transplantation (LT) are one of
the most common complications, occurring in 4.5-32\% of patients. Multiple plastic stenting (MPS)
requires multiple sessions, with the associated risk, cost and patient discomfort. Fully covered self-
expandable metal stents (FC-SEMS) have increasingly been used in this setting. We performed a
systematic review and meta-analysis of randomized controlled trials (RCTs), comparing the role
of FC-SEMS and MPS in the treatment of ABS post-LT.

Methods We conducted a bibliographic search using PubMed and EMBASE, aimed at identifying available RCTs that compared MPS to FC-SEMS in patients with ABS post LT from January 2000 to October 2017. Primary outcomes were ABS resolution and recurrence, while secondary outcomes were adverse events and number of procedures performed. Pooled estimates were calculated using random-effects models.

Results Four RCTs (205 patients) were included. ABS resolution and recurrence did not differ significantly between the groups (odds ratio [OR] 1.05, 95\% confidence interval [CI] 0.43-2.56, $\mathrm{P}=0.92$; and $\mathrm{OR} 2.37,95 \% \mathrm{CI} 0.54-10.38, \mathrm{P}=0.25$ ). The same was true for adverse events (OR 0.91 , $95 \% \mathrm{CI}$ 0.84-3.48, $\mathrm{P}=0.86)$ and migration rate $(\mathrm{OR} 1.31,95 \% \mathrm{CI} 0.46-3.71, \mathrm{P}=0.61)$. The mean number of endoscopic retrograde cholangiopancreatography procedures was lower for FC-SEMS (mean difference [MD] -2.08).

Conclusions FC-SEMS and MPS had equal ABS resolution and recurrence, although there was a trend towards a higher recurrence rate in FC-SEMS that disappeared when trials with a shorter stent indwelling time were excluding. No difference was found in overall adverse events or migration rate.

Keywords Randomized controlled trials, meta-analysis, biliary tract disease, stent, self-expandable metal stent, plastic stent, liver transplantation, anastomotic biliary stricture

Ann Gastroenterol 2019; 32 (4): 1-9

${ }^{a}$ Endoscopy Unit, Ospedale Ca’ Granda Niguarda, Milan, Italy (Alberto Tringali, Giulia Bonato, Marcello Cintolo, Massimiliano Mutignani); bEndoscopy Unit, IRCCS ISMETT, Palermo Italy (Ilaria Tarantino, Luca Barresi, Mario Traina); 'Endoscopy Unit, Nuovo Regina Margherita Hospital, Rome, Italy (Cesare Hassan); ${ }^{\mathrm{D} D i v i s i o n}$ of Gastroenterology \& Hepatology University of Utah, Salt Lake City, Utah, USA (Douglas G. Adler)

Conflict of Interest: none

Correspondence to: Alberto Tringali, MD, Department of Surgery, Endoscopy Unit, Niguarda Hospital, Piazza Ospedale Maggiore 3, 21062 Milan, Italy, e-mail: albtri10@gmail.com; alberto.tringali@ ospedaleniguarda.it

Received 19 January 2019; accepted 23 March 2019; published online 13 April 2019

DOI: https://doi.org/10.20524/aog.2019.0376

๑ 2019 Hellenic Society of Gastroenterology

\section{Introduction}

Anastomotic biliary strictures (ABS) are one of the most common complications following liver transplantation (LT), occurring in approximately $4.5-15 \%$ of cases after deceased donor LT and 8-32\% after living donor-related LT (LDLT) [1-7]. ABS usually develop within the first year after transplant $[1,8,9]$ and their formation is often due to surgical technical issues, a fibroproliferative response to local ischemia, or both [10].

Endoscopic treatment has become the standard of care for the management of ABS and several techniques have been described, including balloon dilation, multiple plastic stent (MPS) insertion and fully covered self-expandable metal stent (FC-SEMS) insertion. Balloon dilation as monotherapy has been abandoned because of its low success rate and high rate of ABS recurrence [11]. 
Currently, the standard of care is the placement of plastic stents with or without balloon dilation, with variable timing of subsequent endoscopic retrograde cholangiopancreatography (ERCP); this approach has proven to be effective and safe, with a low rate of ABS recurrence [12-15]. In order to minimize the issues associated with the placement of multiple plastic stents, such as stent occlusion, suboptimal long-term efficacy and the need for multiple endoscopic sessions, several studies have assessed the validity of single FC-SEMS as an alternative to MPS, with heterogeneous results [16-19].

A previous randomized controlled trial (RCT) comparing different types of FC-SEMS showed a clinical success rate of 70\%, which was below the reported success rate for MPS [20]. This data was confirmed by 3 previous meta-analyses [15,21,22], comparing the efficacy and safety of FC-SEMS vs. MPS in patients with ABS post-LT and LDLT. These meta-analysis, including only observational studies with low quality, showed that, although FC-SEMS was a promising option in this setting, there was no clear advantage of FC-SEMS over MPS. Recently, 2 new RCTs have been published comparing the efficacy and safety of FC-SEMS vs. MPS [23,24].

The aim of this study was to perform an updated systematic review of the current literature comparing the safety and efficacy of FC-SEMS vs. MPS insertion in the management of post-LT ABS.

\section{Patients and methods}

This study was conducted in accordance with the Preferred Reporting Items for Systematic Review and Meta-Analyses (PRISMA) guidelines [25]. The methods of analysis and inclusion criteria were specified in advance and documented in a protocol according to the Cochrane guidelines. A PRISMA checklist is provided in the supplementary materials (S1).

\section{Search strategy}

Studies were identified by searching, with the assistance of a research librarian, in PubMed, EMBASE, Google Scholar and the Cochrane Library. The following MESH and keyword search terms were used: "liver transplantation", "anastomotic biliary stricture", "biliary strictures", "self-expandable metal stents", "plastic stents". Any duplicate citation was removed.

\section{Inclusion criteria}

1. Types of studies: RCTs comparing the efficacy and safety of fully covered SEMS vs. MPS.

2. Types of participants: patients older than 18 years old with ABS following orthotopic LT without previous stent placement.

3. Types of interventions: MPS vs. FC-SEMS placement.

4. Types of outcome measures: ABS resolution and recurrence, cause of stent dysfunction and adverse events.

\section{Exclusion criteria}

Non-randomized studies, non-anastomotic strictures, and other benign biliary strictures were excluded from the meta-analysis. The keywords "biliary tract disease", "biliary obstruction", "biliary stricture", "anastomotic stricture", "liver transplantation", "randomized controlled trial", "meta-analysis", "endoprosthesis", "metal stent", and "systematic review" were associated in different combinations using the Boolean terms AND/OR. Queries were limited to those involving human subjects. Manual searches of reference lists of relevant literature reviews were used to complement the computer searches. A search strategy is provided in the Supplementary Material (S2). Each article was read and analyzed by at least 2 members of the research team (AT \& MM) and eligibility assessment was performed independently in a non-blinded standardized manner.

\section{Definitions}

Only one trial [24] reported definitions of measured outcomes that would allow an objective assessment of the results. ABS was defined as a dominant stricture at the anastomotic site without effective passage of contrast medium, as shown by cholangiographic imaging. ABS resolution was defined as cholangiographic resolution of stricture, assessed by easy passage of an $8.5 \mathrm{~mm}$ extraction balloon through the anastomosis site, and no need for further interventional procedure. ABS recurrence was defined as relapse characterized by the onset of new clinical symptoms and/or increase in cholestatic enzymes or total bilirubin with cholangiographic evidence of an ABS that requires a subsequent interventional procedure. Adverse events were defined as the occurrence of complications after the procedure and graded according to the Cotton criteria [26].

\section{Data collection}

Two investigators (AT \& MC) extracted data from the eligible publications independently. The following data were retrieved into a standardized database:

- Descriptive data: first author, year and type of publication, country of origin, study setting, number of patients, age and sex of patients, reason for LT, time to stricture, treatment time, number of ERCPs, stent type and covering material, MPS protocols, length of follow up, adverse events, and procedural related costs.

- Qualitative data: random sequence generation, allocation concealment, blinding of participant and personnel, blinding outcome assessment, incomplete outcome data, selective reporting, and lost to follow up.

- Outcome data: For primary outcomes we extracted the odds ratio (OR) with $95 \%$ confidence interval (CI), when reported in the original publication, or we collected additional information in order to apply statistical methods 
to compute them. For secondary outcomes, we extracted the number of patients and events in each arm.

- Quality appraisal: Each included study was appraised for quality by 2 independent evaluators (AT \& BG). Quality appraisal was performed using the risk-of-bias tool, as recommended by the Cochrane collaboration [27].

\section{Outcome measures}

The primary outcome measures were the rates of ABS resolution and recurrence. Secondary outcomes included overall adverse events, pancreatitis, cholangitis and bleeding rate, stent migration and the median number of procedures (ERCP). No RCTs reported cost-effectiveness analysis so that these data were not available for statistical analysis.

\section{Statistical analysis}

Dichotomous outcomes were evaluated in terms of ORs with their 95\%CIs and summarized across studies through a random-effects model. If no between-study heterogeneity was evident, the pooled estimate from the random-effects model would be equal to the one derived from a fixed-effects model. Procedure time was analyzed using the Hedges' Standardized Mean Difference estimator. When studies used median and range, results were converted to mean and standard deviation using the formula of Hozo et al [28]. The hazard ratios (HR) for time to achieve resolution and time to re-obstruction could not be obtained because they were not reported in the trials included in the analysis. Finally, we tried to derive the estimation of an indirect measure of the HRs from log-rank P-values or Kaplan-Meier curves, as previously reported [29], but it was only possible to extract the time to reobstruction for 2 trials [23,24] and the time to achieve ABS resolution for one trial [30], not enough to allow the calculation of relevant outcomes. We performed a cost-analysis converting data from one study from Australian to US dollars and calculating median, range and standard deviation comparing using the Student's $t$-test. Between-studies heterogeneity was assessed using the $\mathrm{Q}$ test based on the chi-squared statistics, and inconsistency was quantified in terms of the I2 statistic [31]. In order to assess potential sources of heterogeneity, we firstly performed a sensitivity analysis by removing each study in turn (leaveone-out-method) to evaluate its influence on the final pooled estimate. Publication bias was assessed by visual inspection of funnel plots for asymmetry and through Egger's test for asymmetry [32].

Data were synthesized using Review Manager software (version 5.1 for Windows, the Cochrane Collaboration, Oxford, UK). Finally, we graded the quality of evidence using Grade system software-GRADEpro Guideline Development Tool [Software]. McMaster University, 2015 (developed by Evidence Prime, Inc.) - according to the GRADE recommendation [33].

\section{Results}

\section{Study selection}

Eight hundred fifty unique studies were identified through the systematic review of the literature. Following the screening of abstracts and titles, we identified 103 potentially eligible studies for which full-text reading was required. Finally, 4 studies [23,24,30,34] were included, as shown in the PRISMA flow diagram in Fig. 1.

\section{Characteristics of the included studies}

The main characteristics of the 4 included studies are reported in Table 1. The studies were published between 1995 and 2017 and included a total of 205 patients, randomized to FC-SEMS ( $\mathrm{n}=103)$ or MPS $(\mathrm{n}=102)$.

Two studies $[23,30]$ reported the rate of balloon dilation in FC-SEMS and MPS, showing that FC-SEMS group had a lower rate of balloon dilation before stent placement (OR $0.14,95 \%$ CI $0.06-0.30$ ). One trial [30] did not report the primary and secondary outcomes in the setting of LT because it was designed to compare FC-SEMS and MPS for all benign biliary strictures. We obtained the extracted data from the authors.

In the MPS stent group, the 4 RCTs included in the analysis followed different protocols, as shown in Table 1. No RCTs in the MPS group reported how many patients had ABS resolution at 3-6 or 12 months, preventing a direct comparison between the 2 groups. In the FC-SEMS group, there was different time to stent removal protocols, as shown in Table 1. The evaluation using the Cochrane risk-of-bias tool did not show significant bias (Fig. 2).

\section{Primary outcomes}

Four studies [23,24,30,34], including 103 FC-SEMS patients and 102 MPS patients, reported the ABS resolution rate. There was no statistically significant difference between FC-SEMS and MPS (OR 1.05, 95\%CI 0.43-2.56) (Fig. 3). FCSEMS showed a trend towards a higher recurrence rate, but the difference was not statistically significant (OR 2.37, 95\%CI 0.54-10.38, $\mathrm{P}=0.25 ; I^{2} 53 \%$ ) (Fig. 4).

The sensitivity analysis was performed by removing each trial analysis in turn to assess the influence of each individual study on the global analysis. We noted that excluding the trials in which FC-SEMS were removed later (6 months) $[24,30]$, the trend towards a higher recurrence rate in the FC-SEMS group became more evident (OR 3.90, 95\%CI 0.56-27.25, $\mathrm{P}=0.17$ ), while excluding studies that removed FC-SEMS earlier (12 weeks to 4 months) $[23,34]$ eliminated the difference between the 2 groups (OR 0.89, 95\%CI 0.29-2.79, $\mathrm{P}=0.85$ ). After exclusion of the trial by Martins et al [24], the only one to show a higher recurrence rate for FC-SEMS, no statistically significant difference was noted (OR 1.31, 95\%CI 0.46-3.74, $\mathrm{P}=0.61)$, without heterogeneity $\left(I^{2} 5 \%\right)$. 


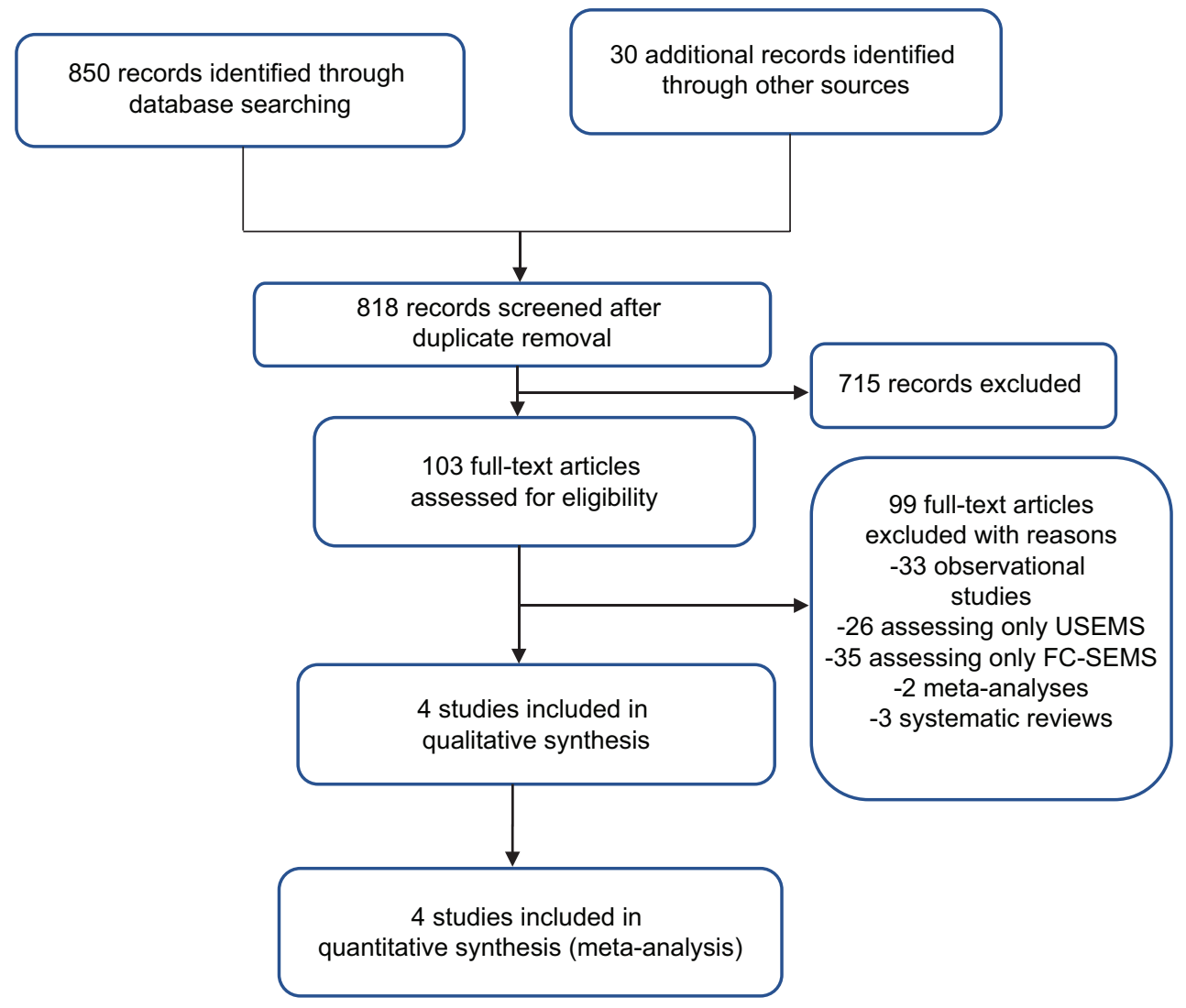

Figure 1 Prisma flow diagram

\section{Secondary outcomes}

The results of the secondary outcomes are provided in Table 2. There were 4 studies, involving 97 FC-SEMS and 89 MPS patients, that reported overall adverse events. The pooled OR was 0.91 (95\%CI 0.32-2.62), showing that there was no statistical difference between FC-SEMS and MPS groups. There was also no statistically significant difference between FC-SEMS and MPS regarding cholangitis rate (OR 0.61, 95\%CI $0.16-2.42, \mathrm{P}=0.49)$, bleeding rate, perforation rate or migration rate.

Acute pancreatitis was assessed in 2 RCTs [24,30] that showed a higher rate of pancreatitis in the FC-SEMS group (OR $3.41,95 \%$ CI $1.16-10.03, \mathrm{P}=0.03$ ). An RCT by Martins et al [24] had a bias due to the fact that the author did not perform endoscopic sphincterotomy before FC-SEMS placement, as highlighted in the interim analysis. When endoscopic sphincterotomy was performed the rate of pancreatitis was equal in both groups.

The median number of ERCP procedures was lower in the FC-SEMS group compared with MPS group (MD -2.08, $95 \%$ CI -3.29 to -0.86$)[23,24,30,34]$. No RCT reported data to allow a cost-effectiveness analysis. Two RCTs [24,34] reported the total costs for both procedures, showing that FC-SEMS allowed a cost saving of between 9,800 and 10,000 US dollars, favoring FC-SEMS as a less expensive procedure $(\mathrm{P}<0.001)$.

A subgroup analysis could not be performed because of the small sample size, insufficient to power the results, and the low number of RCTs published to date. The risk-of-bias analysis of individual studies showed a high risk of performance and detection bias, probably related to the need for ABS assessment, and stent placing and removal by the physicians. The Grade summary of evidence is reported in the Supplementary material (S3).

\section{Publication bias}

Visual inspection of funnel plots (Fig. 5) showed no evidence of asymmetry. As a confirmation, Egger's test for funnel plot asymmetry gave a $\mathrm{P}$-value of 0.13 , showing no potential publication bias for the outcomes considered.

\section{Discussion}

We performed a systematic review and meta-analysis of RCTs specifically designed to assess the efficacy and safety of placement of single FC-SEMS vs. MPS in the management of ABS following LT. In our study, there was no statistically significant difference in the rates of $\mathrm{ABS}$ resolution and recurrence between FC-SEMS and MPS in patients with ABS 
Table 1 Baseline characteristics of included studies

\begin{tabular}{|c|c|c|c|c|c|c|c|c|}
\hline \multirow[t]{2}{*}{ Results } & \multicolumn{2}{|c|}{$\begin{array}{l}\text { Kaffes et al } \\
2014 \text { [34] }\end{array}$} & \multicolumn{2}{|c|}{$\begin{array}{l}\text { Cotè et al } \\
2016[30]\end{array}$} & \multicolumn{2}{|c|}{$\begin{array}{c}\text { Tal et al } \\
2017 \text { [23] }\end{array}$} & \multicolumn{2}{|c|}{$\begin{array}{c}\text { Martins et al } \\
2018 \text { [24] }\end{array}$} \\
\hline & FC-SEMS & MPS & FC-SEMS & MPS & FC-SEMS & MPS & FC-SEMS & MPS \\
\hline $\begin{array}{l}\text { Number of } \\
\text { patients }\end{array}$ & 10 & 10 & 37 & 36 & 24 & 24 & 30 & 29 \\
\hline Age & $56.5(38-67)$ & $49.5(23-69)$ & 55.4 & 56.4 & $57(32-69)$ & $58.5(32.72)$ & $54(23-3)$ & $50(28-71)$ \\
\hline Sex & \multicolumn{2}{|l|}{$5 / 55 / 5$} & \multicolumn{2}{|c|}{$26 / 1125 / 11$} & \multicolumn{2}{|l|}{$14 / 1018 / 6$} & \multicolumn{2}{|l|}{$22 / 820 / 9$} \\
\hline Study type & \multicolumn{2}{|c|}{ RCTs 2 centers } & \multicolumn{2}{|c|}{ RCTs multi } & \multicolumn{2}{|l|}{ RCTs multi } & \multicolumn{2}{|c|}{ RCTs single-center } \\
\hline Stent type & \multicolumn{2}{|l|}{ Taewoong } & \multicolumn{2}{|c|}{ Wallflex $8 / 10 \mathrm{~mm}$} & \multicolumn{2}{|c|}{$\begin{array}{l}3 \text { types without } \\
\text { anti-migration }\end{array}$} & \multicolumn{2}{|c|}{ Wallflex $10 \mathrm{~mm}$} \\
\hline $\begin{array}{l}\text { FC-SEMS removal } \\
\text { (study protocol) }\end{array}$ & \multicolumn{2}{|l|}{12 weeks } & \multicolumn{2}{|l|}{ NA } & \multicolumn{2}{|l|}{ 4-6 months } & \multicolumn{2}{|l|}{6 months } \\
\hline MPS protocol & \multicolumn{2}{|c|}{$\begin{array}{l}\text { Dilation at endoscopist } \\
\text { discretion at } 3 \text { month } \\
\text { remove PS and if stricture } \\
\text { solved not stented }\end{array}$} & \multicolumn{2}{|c|}{$\begin{array}{l}\text { Dilate \& place 1-2 } \\
\text { PS q3 months, } \\
\text { remove all PS, } \\
\text { sequentially dilate } \\
\text { and upsize PS until } \\
\text { stricture solved }\end{array}$} & \multicolumn{2}{|c|}{$\begin{array}{l}\text { As many as possible } \\
\text { of varying diameters } \\
7-10-11.5 \text { and type at } \\
\text { endoscopist discretion }\end{array}$} & \multicolumn{2}{|c|}{$\begin{array}{l}\text { Dilate with balloon } 6-10 \mathrm{~mm} \\
\text { and as many as PS, remove all } \\
\text { PS, dilate restenting until } 12 \\
\text { months }\end{array}$} \\
\hline $\begin{array}{l}\text { No. of ERCPs/ } \\
\text { patients }\end{array}$ & \multicolumn{2}{|c|}{$2(2-2) / 4.5(2-6)$} & \multicolumn{2}{|c|}{$\begin{array}{l}2.21( \pm 0.48) \\
3.13( \pm 0.88)\end{array}$} & \multicolumn{2}{|c|}{$2(2-12) / 4(3-12)$} & \multicolumn{2}{|c|}{$2(2-2) / 4.9(4-6)$} \\
\hline $\begin{array}{l}\text { No. of stents/ } \\
\text { patient }\end{array}$ & \multicolumn{2}{|c|}{$1.0(1-1) / 7.5(3-13)$} & \multicolumn{2}{|l|}{ NA } & \multicolumn{2}{|c|}{$1(1-24) / 8(2-32)$} & \multicolumn{2}{|c|}{$1(1-1) / 16(6-30)$} \\
\hline $\begin{array}{l}\text { Treatment time } \\
\text { median (range) }\end{array}$ & \multicolumn{2}{|c|}{$3.8(2.5-5.0) 10.1(4-13)$} & \multicolumn{2}{|c|}{$\begin{array}{l}158.2( \pm 89.7) \\
193-5( \pm 88.7)\end{array}$} & \multicolumn{2}{|c|}{$\begin{array}{l}178.5(65-551) \\
229.5(59-490)\end{array}$} & \multicolumn{2}{|c|}{$158.5(9-239) / 354(222-242)$} \\
\hline $\begin{array}{l}\text { Balloon dilation } \\
\text { before stenting }\end{array}$ & \multicolumn{2}{|l|}{$0 / 1.5(0-2)$} & $5 / 37$ vs. $31 /$ & & $10 / 24$ vs. 1 & & NA & \\
\hline Costs & $\begin{array}{l}10830 \mathrm{AuD} 2 \\
9674 \text { USD } 21\end{array}$ & $\begin{array}{l}580 \mathrm{AuD} \\
65 \mathrm{USD}\end{array}$ & & & & & 6903 USD & JSD \\
\hline
\end{tabular}

FC-SEMS, fully covered self-expandable metal stents; MPS, multiple plastic stenting; ERCP, endoscopic retrograde cholangiopancreatography; RCT, randomized controlled trial; AuD, Australian dollars; USD, United states dollars

post-LT, although the pooled ORs show that FC-SEMS had a trend for a higher recurrence rate compared with MPS, with between-study heterogeneity (I2 53\%). This trend was especially apparent when stent removal was performed earlier, but the small sample size was not enough to provide robust data that would allow a definitive conclusion. ABS dilation before stent deployment was performed more frequently in the MPS group than in the FC-SEMS group, but it was not possible to assess the effect of biliary dilation on the ABS recurrence rate.

A crucial issue that remains unsolved is ABS recurrence, because more information would be needed. The study by Martins was the only one to show a significantly higher rate of recurrence in the FC-SEMS group, and the authors suggested that the shorter indwelling time (6 months) in the FC-SEMS group was responsible for this result. We suggest according to our data that should be prolonged the indwelling time in the FC-SEMS group and that future RCTs should take this issue into account.

Another critical point to be stressed is the difference between the 2 procedures (FC-SEMS vs. MPS) because, while FC-SEMS is associated with 2 steps (placement and removal after a specified time), the MPS technique involves 5 or 6 procedures (placement and removal). Furthermore, the MPS protocol could be different across different centers and protocols, depending on personal technique, for example, stent removal and replacement, or adding one or more stents without stent removal. In the 4 RCTs included in our metaanalysis, the MPS protocols differed significantly, leading to confounding factors that produced a bias.

One unresolved point that should be analyzed in future trials is the method of FC-SEMS placement (transpapillary vs. intraductal), which would be likely to further influence the performance of FC-SEMS, reducing the migration rate and SEMS dysfunction from sludge. Another point that should be mentioned is the ABS location, because no data was available from the primary studies. This could be a factor that limits the use of FC-SEMS placement, if closeness to the bifurcation causes closure of the duct, resulting in cholangitis.

A meta-analysis by Kao et al including only observational studies, assessed the role of MPS in 8 studies (446 patients) in CDLT, 3 studies (120 patients) using MPS in LDLT, and 

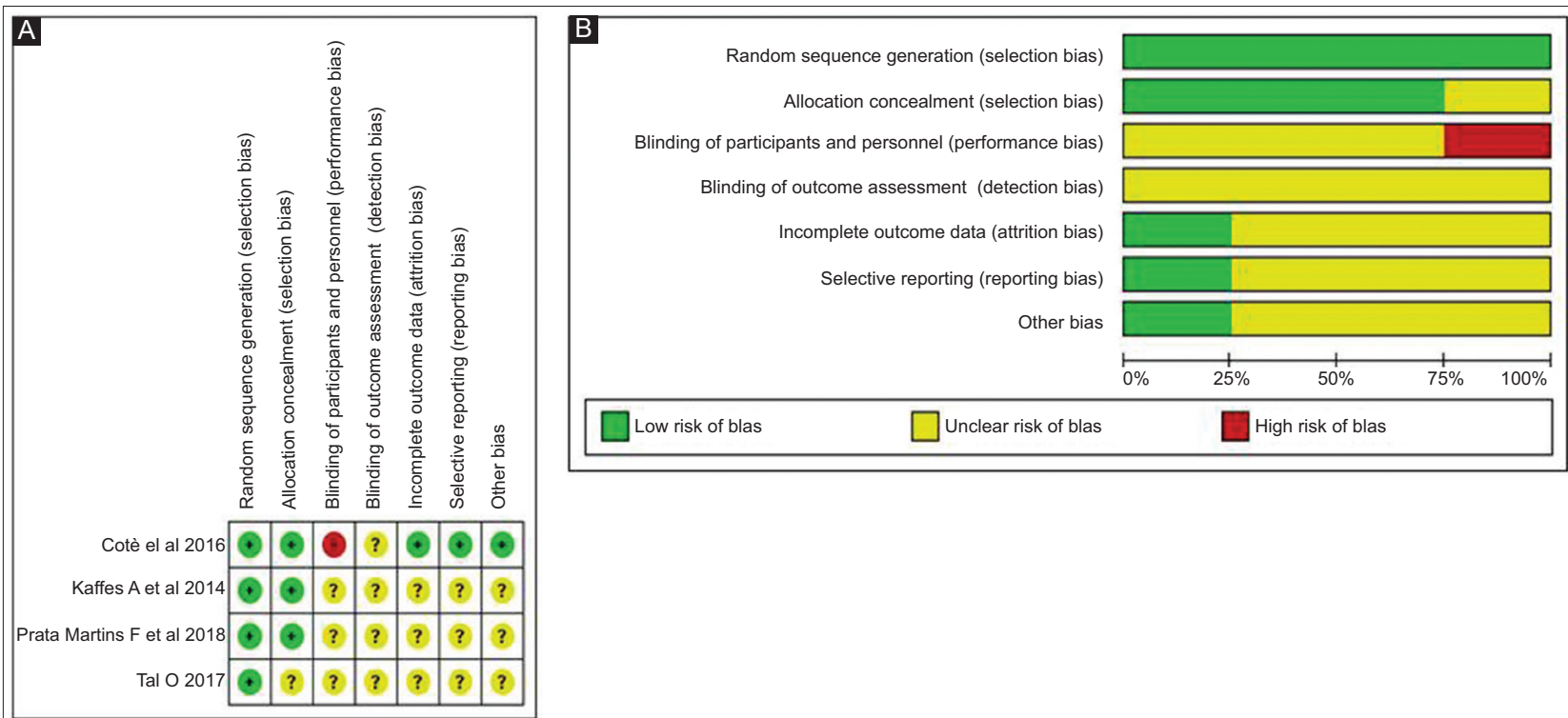

Figure 2 Cochrane risk of bias. (A) Risk of bias summary. (B) risk of bias in the individual study

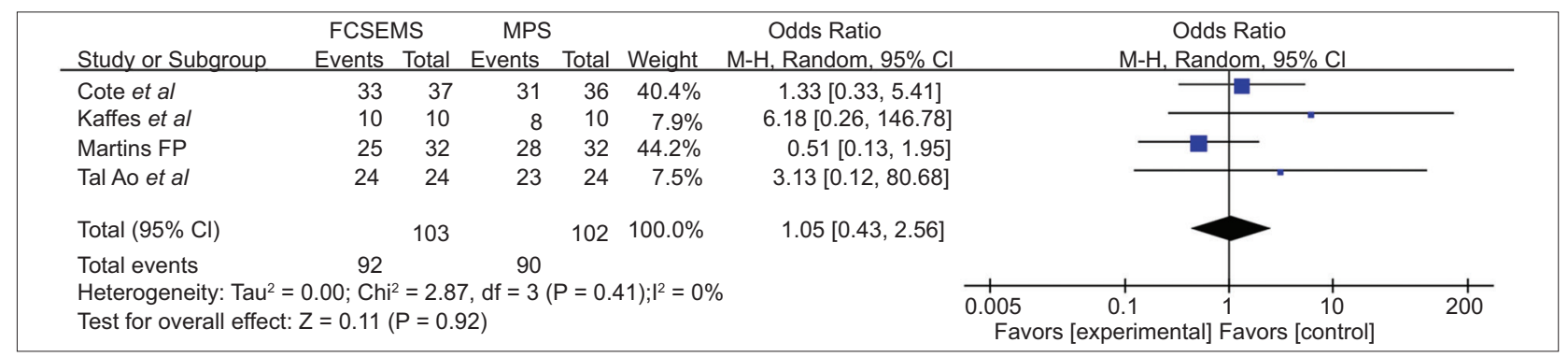

Figure 3 Forest plots showing the results of a meta-analysis comparing stricture resolution between FC-SEMS and MPS CI, confidence interval; FC-SEMS, fully covered self-expandable metal stents; MPS, multiple plastic stenting

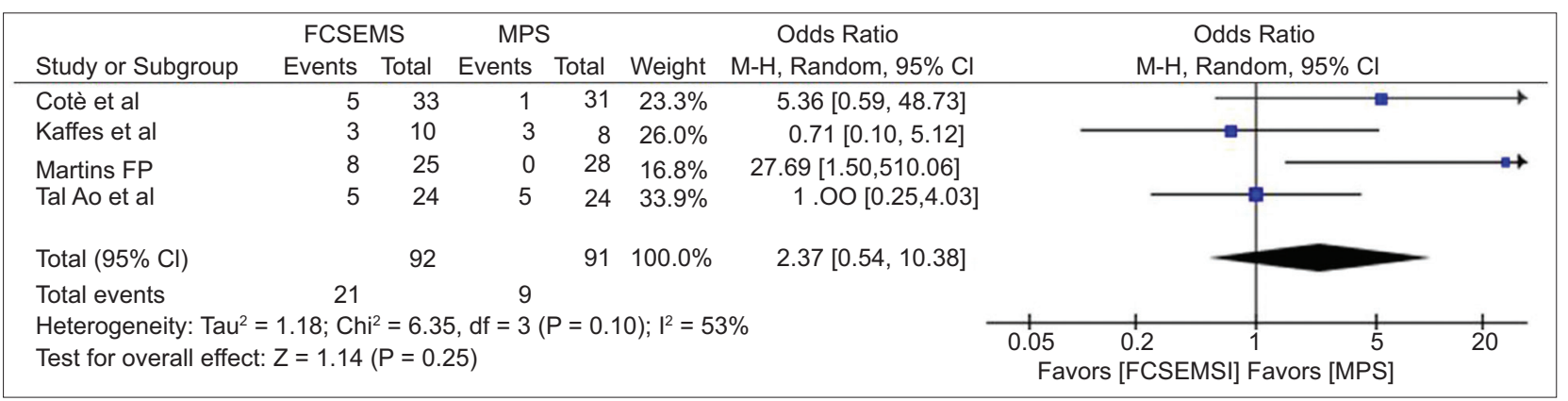

Figure 4 Forest-plots showing the results of meta-analysis comparing stricture recurrence between FC-SEMS vs. MPS

CI, confidence interval; FC-SEMS, fully covered self-expandable metal stents; MPS, multiple plastic stenting

10 studies (200 patients) using SEMSs without a direct comparing of the two treatments. The authors concluded that $\mathrm{ABS}$ resolution and recurrence rate were higher in cases with a longer stent indwell duration (>12 months) compared with a shorter duration ( $<12$ months); this is similar to our own findings.

In the meta-analysis by Kao et al [15] available data were in the form of case series. Each study had small numbers of patients, with 148 being the highest number of participants, and none fulfilled all the criteria for high-quality studies. Significant heterogeneity existed among the studies with respect to primary outcome, patient selection, stent protocol, stent duration, types of SEMS and follow-up periods; this made it difficult to compare FC-SEMS with MPS.

A meta-analysis by Aparicio et al [21] that included 10 studies (1 RCT, 6 non-randomized prospective studies and 3 cohort studies) showed an equal ABS resolution with FC-SEMS and MPS, but only the single RCT assessed the 
Table 2 Primary and secondary outcome

\begin{tabular}{|c|c|c|c|c|c|c|c|c|}
\hline Author & $\begin{array}{c}\text { Total n. } \\
\text { pts }\end{array}$ & $\begin{array}{l}\text { FC-SEMS/ } \\
\text { MPS }\end{array}$ & $\begin{array}{c}\text { Stricture } \\
\text { resolution } \\
(\mathrm{n} / \%) \\
\text { FC-SEMS/MPS }\end{array}$ & $\begin{array}{c}\text { Stricture } \\
\text { recurrence } \\
(\mathrm{n} / \%) \\
\text { FC-SEMS/MPS }\end{array}$ & $\begin{array}{c}\text { Overall } \\
\text { adverse events } \\
(\mathrm{n} / \%) \\
\text { FC-SEMS/MPS }\end{array}$ & $\begin{array}{c}\text { Migration rate } \\
(\mathrm{n} / \%) \\
\text { FC-SEMS/MPS }\end{array}$ & $\begin{array}{c}\text { Acute } \\
\text { pancreatitis } \\
(\mathrm{n} / \%) \\
\text { FC-SEMS/MPS }\end{array}$ & $\begin{array}{l}\text { Cholangitis rate } \\
\qquad(\mathrm{n} / \%) \\
\text { FC-SEMS vs } \\
\text { MPS }\end{array}$ \\
\hline $\begin{array}{l}\text { Martin et al, } \\
2017 \text { [24] }\end{array}$ & 59 & $30 / 29$ & $25 / 30$ vs. $28 / 29$ & $8 / 25$ vs. $0 / 28$ & $14 / 30$ vs. $9 / 30$ & $3 / 30$ vs. $4 / 30$ & $8 / 60$ vs. $3 / 141$ & $1 / 60$ vs. $2 / 141$ \\
\hline $\begin{array}{l}\text { Tal et al, } \\
2017 \text { [23] }\end{array}$ & 48 & $24 / 24$ & $24 / 24$ vs. $23 / 24$ & $5 / 24$ vs. $5 / 24$ & $3 / 24$ vs. $3 / 24$ & $5 / 24$ vs. $0 / 24$ & NA & NA \\
\hline $\begin{array}{l}\text { Kaffes et al, } \\
2014 \text { [33] }\end{array}$ & 20 & $10 / 10$ & $10 / 10$ vs. $8 / 10$ & $3 / 10$ vs. $3 / 8$ & $6 / 10$ vs. $5 / 10$ & $0 / 10$ vs. $1 / 10$ & NA & $1 / 10$ vs. $4 / 10$ \\
\hline $\begin{array}{l}\text { Cote et al, } \\
2016[30]\end{array}$ & 73 & $37 / 36$ & $33 / 37$ vs. $31 / 36$ & $5 / 33$ vs. $1 / 31$ & $12 / 33$ vs. $17 / 25$ & $15 / 33$ vs. $8 / 25$ & $2 / 33$ vs. $2 / 25$ & $1 / 33$ vs. $0 / 25$ \\
\hline Overall & 205 & $103 / 102$ & $92 / 103$ vs. $90 / 102$ & $21 / 92$ vs. $9 / 91$ & $35 / 97$ vs. $34 / 89$ & $23 / 101$ vs. $13 / 89$ & $10 / 93$ vs. $5 / 166$ & $3 / 103$ vs. $6 / 176$ \\
\hline $\begin{array}{l}\text { OR }(95 \% \mathrm{CI}) \\
\mathrm{P} \text { value }\end{array}$ & & & $\begin{array}{c}1.05(0.43,2.56) \\
0.92\end{array}$ & $\begin{array}{c}2.37(0.54-10.38) \\
0.25\end{array}$ & $\begin{array}{c}0.91(0.84-3.48) \\
0.86\end{array}$ & $\begin{array}{c}1.31(0.46-3.71) \\
0.61\end{array}$ & $\begin{array}{c}3.41(1.16-10.03) \\
0.03\end{array}$ & $\begin{array}{c}0.61(0.16-2.42) \\
0.49\end{array}$ \\
\hline
\end{tabular}

FC-SEMS, fully covered self-expandable metal stents; MPS, multiple plastic stenting; OR, odds ratio; CI, confidence interval

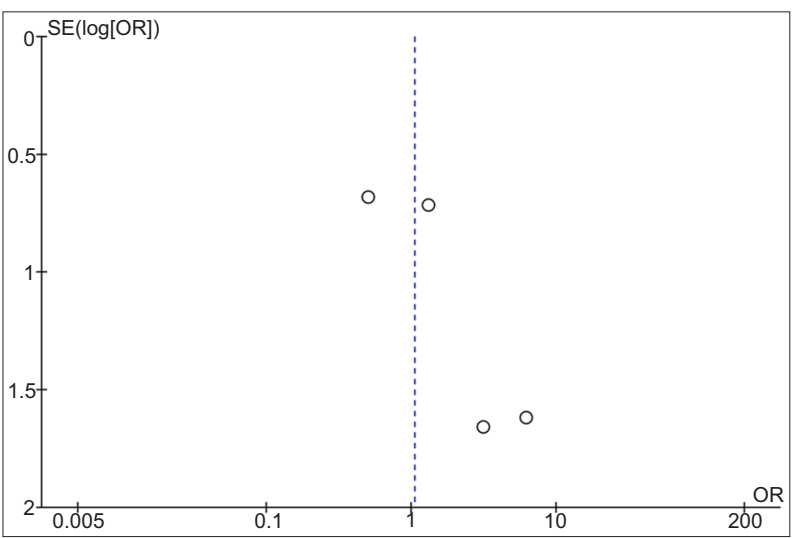

Figure 5 Funnel plot showing no publication bias SE, standard error; OR, odds ratio

ABS resolution rate between FC-SEMS and MPS. A recent meta-analysis was published by Landi et al [22], including 3 RCTs and 1 observational study with a total of 119 patients receiving FC-SEMS and 179 MPS. The results showed that FC-SEMS were superior in terms of the number of ERCP procedures and days of treatment, whereas ABS resolution and recurrence rate showed equal efficacy in both groups. This meta-analysis is underpowered to draw definitive conclusions and the consistent heterogeneity observed in the included studies suggest caution for interpreting the results. The authors did not consider the results of previous metaanalyses, while the findings regarding ABS resolution and recurrence from the randomized trials by Cote et al [30], designed for all benign biliary strictures, included in their meta-analysis, did not report the real results for the subgroup of ABS post-LT.

Only 2 RCTs, those by Kaffes et al [34] and Martins et al [24], reported the cost-analysis, which included the cost of the procedure and the health personnel involved, and both showed a cost-saving favoring FC-SEMS. Furthermore, an economic analysis published in abstract form found that FC-SEMS were overwhelming favored as the more economical strategy, offering an overall less expensive hospital stay and fewer lost days of work compared to MPS, even with the more expensive implant cost.

The limitations of our meta-analysis are based on the missing relevant data from the primary studies, such as risk factors for biliary complications, different protocols, need for balloon dilation, SEMS type, type of covering, different axial and radial force, and the different protocols used in the RCTs for the MPS group. Another limitation is the absence of costanalysis and cost-benefit studies that could allow a choice of better treatment approach, taking into account the costs to the health system. The small sample size (205 patients) of the RCTs included could have affected various results and limited the conclusions; it did not allow us to determine the reasons for the trend noted towards a higher recurrence rate in the FC-SEMS group. Finally, we assessed ABS resolution and recurrence as a dichotomous variable and not as a time to event, because, unfortunately, the HRs of the 2 primary outcomes were often unavailable or not obtainable.

In conclusion, our systematic review shows that FC-SEMS had equal stricture resolution, stricture recurrence and overall adverse events compared with MPS, although there was a trend toward a higher recurrence rate in the FC-SEMS groups when the stents were removed early. Our meta-analysis is the first systematic review including only RCTs that have conducted to a more robust conclusion. FC-SEMS are associated with reduced number of procedures overall a cost sparing procedure. According to our data and cost-analysis data, the use of new FC-SEMS with an antimigration system for a longer dwell time could be the best approach. Further RCTs with larger sample size and longer follow up focusing on risk factors of recurrence rate and using FC-SEMS with anti-migration features with appropriate dwelling time are warranted. 


\section{Acknowledgment}

We thank the data providers, Stuart Sherman, MD, Indiana University School of Medicine, Gastroenterology, \& Leslie J. Pfeffer, BS, CHP University HIPAA Privacy, for permission to use de-identified data ("Summary of the data collected for this study from patients with post-liver transplantation biliary stricture") for the purposes of this article.

\section{Summary Box}

\section{What is already known:}

- Multiple plastic stents (MPS) are the gold standard for the treatment of anastomotic biliary strictures post-liver transplantation

- Fully covered self-expandable metal stents (FCSEMS) are increasingly used as rescue treatment in case of refractory strictures or as primary treatment

- Many trials and meta-analyses addressed the comparison between MPS and FC-SEMS without finding any relevant difference, but those studies had many flaws

\section{What the new findings are:}

- Our study for the first time analyzed only randomized controlled trials (RCTs) and showed that MPS and FC-SEMS are equally effective and safe procedures

- We stressed the difference between the 2 procedures because, while FC-SEMS is associated with 2 steps (placement and removal after a specific time), the MPS technique involves 5 or 6 steps (placement and removal)

- The study highlights the missing relevant data from the primary studies, such as risk factors for biliary complications, different protocols, need for balloon dilation, SEMS type, type of covering, different axial and radial forces, the different protocols used in the RCTs, as well as the absence of cost-analysis that could allow a choice of better treatment approach considering health system costs

\section{References}

1. Greif F, Bronsther OL, Van Thiel DH, et al. The incidence, timing, and management of biliary tract complications after orthotopic liver transplantation. Ann Surg 1994;219:40-45.

2. Thethy S, Thomson BN, Pleass H, et al. Management of biliary tract complications after orthotopic liver transplantation. Clin Transplant 2004;18:647-653.

3. Rerknimitr $\mathrm{R}$, Sherman $\mathrm{S}$, Fogel EL, et al. Biliary tract complications after orthotopic liver transplantation with choledochocholedochostomy anastomosis: endoscopic findings and results of therapy. Gastrointest Endosc 2002;55:224-231.

4. Gondolesi GE, Varotti G, Florman SS, et al. Biliary complications in 96 consecutive right lobe living donor transplant recipients. Transplantation 2004;77:1842-1848.

5. Soejima Y, Taketomi A, Yoshizumi T, et al. Biliary strictures in living donor liver transplantation: incidence, management, and technical evolution. Liver Transpl 2006;12:979-986.

6. Chang JH, Lee I, Choi MG, Han SW. Current diagnosis and treatment of benign biliary strictures after living donor liver transplantation. World J Gastroenterol 2016;22:1593-1606.

7. Sharma S, Gurakar A, Jabbour N. Biliary strictures following liver transplantation: past, present and preventive strategies. Liver Transpl 2008;14:759-769.

8. Albert JG, Filmann N, Elsner J, et al. Long-term follow-up of endoscopic therapy for stenosis of the biliobiliary anastomosis associated with orthotopic liver transplantation. Liver Transpl 2013; 19:586-593.

9. Verdonk RC, Buis CI, Porte RJ, et al. Anastomotic biliary strictures after liver transplantation: causes and consequences. Liver Transpl 2006;12:726-735.

10. Koksal AS, Eminler AT, Parlak E, Gurakar A. Management of biliary anastomotic strictures after liver transplantation. Transplant Rev (Orlando) 2017;31:207-217.

11. Zoepf T, Maldonado-Lopez EJ, Hilgard P, et al. Balloon dilatation vs. balloon dilatation plus bile duct endoprostheses for treatment of anastomotic biliary strictures after liver transplantation. Liver Transpl 2006;12:88-94.

12. Tringali A, Barbaro F, Pizzicannella M, et al. Endoscopic management with multiple plastic stents of anastomotic biliary stricture following liver transplantation: long-term results. Endoscopy 2016;48:546-551.

13. Tabibian JH, Asham EH, Han S, et al. Endoscopic treatment of postorthotopic liver transplantation anastomotic biliary strictures with maximal stent therapy (with video). Gastrointest Endosc 2010;71:505-512.

14. Costamagna G, Pandolfi M, Mutignani M, Spada C, Perri V. Longterm results of endoscopic management of postoperative bile duct strictures with increasing numbers of stents. Gastrointest Endosc 2001;54:162-168.

15. Kao D, Zepeda-Gomez S, Tandon P, Bain VG. Managing the post-liver transplantation anastomotic biliary stricture: multiple plastic versus metal stents: a systematic review. Gastrointest Endosc 2013;77:679-691.

16. Chaput U, Scatton O, Bichard P, et al. Temporary placement of partially covered self-expandable metal stents for anastomotic biliary strictures after liver transplantation: a prospective, multicenter study. Gastrointest Endosc 2010;72:1167-1174.

17. Tarantino I, Traina M, Mocciaro F, et al. Fully covered metallic stents in biliary stenosis after orthotopic liver transplantation. Endoscopy 2012;44:246-250.

18. Sauer P, Chahoud F, Gotthardt D, et al. Temporary placement of fully covered self-expandable metal stents in biliary complications after liver transplantation. Endoscopy 2012;44:536-538.

19. Hu B, Gao DJ, Yu FH, Wang TT, Pan YM, Yang XM. Endoscopic stenting for post-transplant biliary stricture: usefulness of a novel removable covered metal stent. J Hepatobiliary Pancreat Sci 2011;18:640-645.

20. Cerecedo-Rodriguez J, Phillips M, Figueroa-Barojas P, et al. Self expandable metal stents for anastomotic stricture following liver transplant. Dig Dis Sci 2013;58:2661-2666.

21. Aparicio DPDS, Otoch JP, Montero EFS, Khan MA, Artifon ELA. Endoscopic approach for management of biliary strictures in liver transplant recipients: A systematic review and meta-analysis. United European Gastroenterol J 2017;5:827-845.

22. Landi F, de'Angelis N, Sepulveda A, et al. Endoscopic treatment of anastomotic biliary stricture after adult deceased donor liver 
transplantation with multiple plastic stents versus self-expandable metal stents: a systematic review and meta-analysis. Transpl Int 2018;31:131-151.

23. Tal AO, Finkelmeier F, Filmann N, et al. Multiple plastic stents versus covered metal stent for treatment of anastomotic biliary strictures after liver transplantation: a prospective, randomized, multicenter trial. Gastrointest Endosc 2017;86:1038-1045.

24. Martins FP, De Paulo GA, Contini MLC, Ferrari AP. Metal versus plastic stents for anastomotic biliary strictures after liver transplantation: a randomized controlled trial. Gastrointest Endosc 2018;87:131.e1-131.e13.

25. Moher D, Liberati A, Tetzlaff J, Altman DG; PRISMA Group. Preferred reporting items for systematic reviews and metaanalyses: the PRISMA statement. PLoS Med 2009;6:e1000097.

26. Cotton PB, Lehman G, Vennes J, et al. Endoscopic sphincterotomy complications and their management: an attempt at consensus. Gastrointest Endosc 1991;37:383-393.

27. Higgins JP, Altman DG, Gøtzsche PC, et al; Cochrane Statistical Methods Group. The Cochrane Collaboration's tool for assessing risk of bias in randomised trials. BMJ 2011;343:d5928.
28. Hozo SP, Djulbegovic B, Hozo I. Estimating the mean and variance from the median, range, and the size of a sample. BMC Med Res Methodol 2005;5:13.

29. Tierney JF, Stewart LA, Ghersi D, Burdett S, Sydes MR. Practical methods for incorporating summary time-to-event data into metaanalysis. Trials 2007;8:16.

30. Coté GA, Slivka A, Tarnasky P, et al. Effect of covered metallic stents compared with plastic stents on benign biliary stricture resolution: A randomized clinical trial. JAMA 2016;315:1250-1257.

31. Higgins JP, Thompson SG. Quantifying heterogeneity in a metaanalysis. Stat Med 2002;21:1539-1558.

32. Egger M, Davey Smith G, Schneider M, Minder C. Bias in metaanalysis detected by a simple, graphical test. BMJ 1997;315:629-634.

33. Schünemann H, Brożek J, Guyatt G OA. GRADE handbook for grading quality of evidence and strength of recommendations. Updated October 2013. The GRADE Working Group, 2013. 2013.

34. Kaffes A, Griffin S, Vaughan R, et al. A randomized trial of a fully covered self-expandable metallic stent versus plastic stents in anastomotic biliary strictures after liver transplantation. Therap Adv Gastroenterol 2014;7:64-71. 


\section{Supplementary material}

Supplementary Table 1 PRISMA check list

PRISMA 2009 Checklist to be included with meta-analyses

\begin{tabular}{|c|c|c|c|}
\hline Section/topic & Item \# & Checklist item & $\begin{array}{l}\text { Reported } \\
\text { on page \# }\end{array}$ \\
\hline \multicolumn{4}{|l|}{ Title } \\
\hline Title & 1 & Identify the report as a systematic review, meta-analysis, or both & $1-3$ \\
\hline \multicolumn{4}{|l|}{ Abstract } \\
\hline Structured summary & 2 & $\begin{array}{l}\text { Provide a structured summary including, as applicable, background; objectives; } \\
\text { data sources; study eligibility criteria, participants, and interventions; study } \\
\text { appraisal and synthesis methods; results; limitations; conclusions and } \\
\text { implications of key findings; systematic review registration number }\end{array}$ & 2 \\
\hline \multicolumn{4}{|l|}{ Introduction } \\
\hline Rationale & 3 & Describe the rationale for the review in the context of what is already known & 3 \\
\hline Objectives & 4 & $\begin{array}{l}\text { Provide an explicit statement of questions being addressed with reference to } \\
\text { participants, interventions, comparisons, outcomes, and study design (PICOS) }\end{array}$ & 3 \\
\hline \multicolumn{4}{|l|}{ Methods } \\
\hline Protocol and registration & 5 & $\begin{array}{l}\text { Indicate if a review protocol exists, if and where it can be accessed (e.g., Web } \\
\text { address), and, if available, provide registration information including registration } \\
\text { number }\end{array}$ & 4 \\
\hline Eligibility criteria & 6 & $\begin{array}{l}\text { Specify study characteristics (e.g., PICOS, length of follow up) and report } \\
\text { characteristics (e.g., years considered, language, publication status) used as } \\
\text { criteria for eligibility, giving rationale }\end{array}$ & 4 \\
\hline Information sources & 7 & $\begin{array}{l}\text { Describe all information sources (e.g., databases with dates of coverage, contact with } \\
\text { study authors to identify additional studies) in the search and date last searched }\end{array}$ & 4 \\
\hline Search & 8 & $\begin{array}{l}\text { Present full electronic search strategy for at least one database, including any } \\
\text { limits used, such that it could be repeated }\end{array}$ & $4-5$ \\
\hline Study selection & 9 & $\begin{array}{l}\text { State the process for selecting studies (i.e., screening, eligibility, included in } \\
\text { systematic review, and, if applicable, included in the meta-analysis) }\end{array}$ & 6 \\
\hline Data collection process & 10 & $\begin{array}{l}\text { Describe method of data extraction from reports (e.g., piloted forms, } \\
\text { independently, in duplicate) and any processes for obtaining and confirming } \\
\text { data from investigators }\end{array}$ & 6 \\
\hline Data items & 11 & $\begin{array}{l}\text { List and define all variables for which data were sought (e.g., PICOS, funding } \\
\text { sources) and any assumptions and simplifications made }\end{array}$ & 6 \\
\hline Risk of bias in individual studies & 12 & $\begin{array}{l}\text { Describe methods used for assessing risk of bias of individual studies (including } \\
\text { specification of whether this was done at the study or outcome level), and how } \\
\text { this information is to be used in any data synthesis }\end{array}$ & 6 \\
\hline Summary measures & 13 & State the principal summary measures (e.g., risk ratio, difference in means) & $6-7$ \\
\hline Synthesis of results & 14 & $\begin{array}{l}\text { Describe the methods of handling data and combining results of studies, if done, } \\
\text { including measures of consistency (e.g., I2) for each meta-analysis }\end{array}$ & 7 \\
\hline Risk of bias across studies & 15 & $\begin{array}{l}\text { Specify any assessment of risk of bias that may affect the cumulative } \\
\text { evidence (e.g., publication bias, selective reporting within studies) }\end{array}$ & 7 \\
\hline Additional analyses & 16 & $\begin{array}{l}\text { Describe methods of additional analyses (e.g., sensitivity or subgroup analyses, } \\
\text { meta-regression), if done, indicating which were pre-specified }\end{array}$ & 7 \\
\hline \multicolumn{4}{|l|}{ Results } \\
\hline Study selection & 17 & $\begin{array}{l}\text { Give numbers of studies screened, assessed for eligibility, and included in the } \\
\text { review, with reasons for exclusions at each stage, ideally with a flow diagram }\end{array}$ & 8 \\
\hline Study characteristics & 18 & $\begin{array}{l}\text { For each study, present characteristics for which data were extracted (e.g., study } \\
\text { size, PICOS, follow-up period) and provide the citations }\end{array}$ & 8 \\
\hline Risk of bias within studies & 19 & $\begin{array}{l}\text { Present data on risk of bias of each study and, if available, any outcome level } \\
\text { assessment (see item 12) }\end{array}$ & 9 \\
\hline
\end{tabular}


Supplementary Table 1 (Continued)

\begin{tabular}{|c|c|c|c|}
\hline Section/topic & Item \# & Checklist item & $\begin{array}{l}\text { Reported } \\
\text { on page \# }\end{array}$ \\
\hline Results of individual studies & 20 & $\begin{array}{l}\text { For all outcomes considered (benefits or harms), present, for each study: (a) } \\
\text { simple summary data for each intervention group and (b) effect estimates and } \\
\text { confidence intervals, ideally with a forest plot }\end{array}$ & 9 \\
\hline Synthesis of results & 21 & $\begin{array}{l}\text { Present the main results of the review. If meta-analyses are done, include } \\
\text { confidence intervals and measures of consistency for each }\end{array}$ & 9 \\
\hline Risk of bias across studies & 22 & Present results of any assessment of risk of bias across studies (see item 15) & 9 \\
\hline Additional analysis & 23 & $\begin{array}{l}\text { Give results of additional analyses, if done (e.g., sensitivity or subgroup analyses, } \\
\text { meta-regression [see item 16]) }\end{array}$ & $9-10$ \\
\hline \multicolumn{4}{|l|}{ Discussion } \\
\hline Summary of evidence & 24 & $\begin{array}{l}\text { Summarize the main findings including the strength of evidence for each main } \\
\text { outcome: consider their relevance to key groups (e.g., healthcare providers, } \\
\text { users, and policy makers) }\end{array}$ & $10-11$ \\
\hline Limitations & 25 & $\begin{array}{l}\text { Discuss limitations at study and outcome level (e.g., risk of bias), and at } \\
\text { review-level (e.g., incomplete retrieval of identified research, reporting bias) }\end{array}$ & 12 \\
\hline Conclusions & 26 & $\begin{array}{l}\text { Provide a general interpretation of the results in the context of other evidence, } \\
\text { and implications for future research }\end{array}$ & 12 \\
\hline \multicolumn{4}{|l|}{ Funding } \\
\hline Funding & 27 & $\begin{array}{l}\text { Describe sources of funding for the systematic review and other support (e.g., } \\
\text { supply of data); role of funders for the systematic review }\end{array}$ & 12 \\
\hline
\end{tabular}

\section{Search strategy}

\section{Supplementary material 1}

\section{Search strategy PubMed}

1. Biliary stricture (exp)

2. Bile duct disease

3. Cholestasis

4. Common bile duct disease

5. Jaundice, obstructive

5. Or/ 1-5

6. Liver transplantation

7. OLT

8. Or/6-7

9. Stent

10. Plastic stent

11. Or/ 9-10

12. Metallic stent

13. SEMS

14. Self-expandable metal stent

15. Or/12-14

16. Randomized controlled trial (pt)

17. Controlled clinical trial

18. Randomized (tiab)

19. Randomly (tiab)

20. Trial (tiab)

21. Groups (tiab)

22. Or/16-21

23. 5 and 8 and 11 and 15 and 22 


\section{Grade summary of evidence}

[FC-SEMS] compared to [MPS] for anastomotic biliary stricture (ABS) post-LT

Patient or population: Anastomotic biliary stricture (ABS) post LT

Setting: Liver transplantation

Intervention: [FC-SEMS]

Comparison: [MPS]

\begin{tabular}{|c|c|c|c|c|c|}
\hline \multirow[t]{2}{*}{ Outcomes } & \multirow{2}{*}{$\begin{array}{l}\text { № of } \\
\text { participants } \\
\text { (studies) } \\
\text { Follow up }\end{array}$} & \multirow{2}{*}{$\begin{array}{l}\text { Certainty of } \\
\text { the evidence } \\
\text { (GRADE) }\end{array}$} & \multirow{2}{*}{$\begin{array}{l}\text { Relative effect } \\
\quad(95 \% \mathrm{CI})\end{array}$} & \multicolumn{2}{|c|}{ Anticipated absolute effects } \\
\hline & & & & Risk with [MPS] & $\begin{array}{l}\text { Risk difference } \\
\text { with [FC-SEMS] }\end{array}$ \\
\hline Stricture resolution & $\begin{array}{c}205 \\
\text { (4 RCTs) }\end{array}$ & $\begin{array}{l}\oplus \oplus \oplus \oplus \\
\mathrm{HIGH}\end{array}$ & $\begin{array}{c}\text { OR } 1.05 \\
(0.43 \text { to } 2.56)\end{array}$ & 88 per 100 & $\begin{array}{l}0 \text { fewer per } 100 \\
\text { (12 fewer to } 7 \text { more) }\end{array}$ \\
\hline Stricture recurrence & $\begin{array}{c}183 \\
(4 \mathrm{RCTs})\end{array}$ & $\begin{array}{l}\oplus \oplus \oplus \oplus \\
\mathrm{HIGH}\end{array}$ & $\begin{array}{c}\text { OR } 2.37 \\
(0.54 \text { to } 10.38)\end{array}$ & 10 per 100 & $\begin{array}{l}11 \text { more per } 100 \\
\text { ( } 4 \text { fewer to } 43 \text { more) }\end{array}$ \\
\hline Adverse events & $\begin{array}{c}186 \\
(4 \mathrm{RCTs})\end{array}$ & $\begin{array}{l}\oplus \oplus \oplus \oplus \\
\mathrm{HIGH}\end{array}$ & $\begin{array}{c}\text { OR } 0.91 \\
(0.84 \text { to } 3.48)\end{array}$ & 38 per 100 & $\begin{array}{l}2 \text { fewer per } 100 \\
\text { (4 fewer to } 30 \text { more) }\end{array}$ \\
\hline Stent migration & $\begin{array}{c}190 \\
(4 \mathrm{RCTs})\end{array}$ & $\begin{array}{l}\oplus \oplus \oplus \oplus \\
\mathrm{HIGH}\end{array}$ & $\begin{array}{c}\text { OR } 1.31 \\
(0.46 \text { to } 3.71)\end{array}$ & 15 per 100 & $\begin{array}{l}4 \text { more per } 100 \\
(7 \text { fewer to } 24 \text { more) }\end{array}$ \\
\hline $\begin{array}{l}\text { Treatment time } \\
\text { assessed with: days }\end{array}$ & $\begin{array}{c}203 \\
\text { (4 RCTs) }\end{array}$ & $\begin{array}{l}\oplus \oplus \oplus \oplus \\
\text { MODERATE }^{a}\end{array}$ & - & $\begin{array}{c}\text { The mean treatment } \\
\text { time was } 266.7\end{array}$ & $\begin{array}{l}\text { MD } 104.99 \text { lower } \\
\text { (202.19 lower to } 7.79 \text { lower) }\end{array}$ \\
\hline Cholangitis & $\begin{array}{c}279 \\
\text { (3 RCTs) }\end{array}$ & $\begin{array}{l}\oplus \oplus \oplus \oplus \\
\mathrm{HIGH}\end{array}$ & $\begin{array}{c}\text { OR } 0.61 \\
(0.16 \text { to } 2.42)\end{array}$ & 34 per 1,000 & $\begin{array}{l}13 \text { fewer per } 1,000 \\
\text { ( } 28 \text { fewer to } 45 \text { more) }\end{array}$ \\
\hline Acute pancreatitis & $\begin{array}{c}259 \\
\text { (3 RCTs) }\end{array}$ & $\begin{array}{l}\oplus \oplus \oplus \oplus \\
\text { MODERATE }\end{array}$ & $\begin{array}{c}\text { OR } 3.41 \\
\text { (1.16 to } 10.03)\end{array}$ & 30 per 1,000 & $\begin{array}{l}66 \text { more per } 1,000 \\
\text { ( } 5 \text { more to } 207 \text { more) }\end{array}$ \\
\hline
\end{tabular}

${ }^{\star}$ The risk in the intervention group (and its $95 \%$ confidence interval) is based on the assumed risk in the comparison group and the relative effect of the intervention (and its 95\%CI)

FC-SEMS, fully covered self-expandable metal stents; MPS, multiple plastic stenting; LT, liver transplantation; CI, confidence interval; OR, odds ratio; MD, mean difference; RCT, randomized controlled trial

GRADE Working Group grades of evidence

High certainty: We are very confident that the true effect lies close to that of the estimate of the effect

Moderate certainty: We are moderately confident in the effect estimate: The true effect is likely to be close to the estimate of the effect, but there is a possibility that it is substantially different

Low certainty: Our confidence in the effect estimate is limited: The true effect may be substantially different from the estimate of the effect

Very low certainty: We have very little confidence in the effect estimate: The true effect is likely to be substantially different from the estimate of effect 